Research Article

\title{
A Study of Fourth-Order Hankel Determinants for Starlike Functions Connected with the Sine Function
}

\author{
Hai-Yan Zhang and Huo Tang $\mathbb{D}$ \\ School of Mathematics and Computer Sciences, Chifeng University, Chifeng 024000, China \\ Correspondence should be addressed to Huo Tang; thth2009@163.com
}

Received 11 March 2021; Revised 9 April 2021; Accepted 16 April 2021; Published 24 April 2021

Academic Editor: Teodor Bulboaca

Copyright (c) 2021 Hai-Yan Zhang and Huo Tang. This is an open access article distributed under the Creative Commons Attribution License, which permits unrestricted use, distribution, and reproduction in any medium, provided the original work is properly cited.

In this paper, upper bounds for the fourth-order Hankel determinant $H_{4}(1)$ for the function class $\mathcal{S}_{s}^{*}$ associated with the sine function are given.

\section{Introduction}

Let $\mathscr{A}$ denote the class of functions $f$ which are analytic in the open unit disk $\mathbb{D}=\{z:|z|<1\}$ of the form

$$
f(z)=z+a_{2} z^{2}+a_{3} z^{3}+\cdots(z \in \mathbb{D})
$$

and let $\mathcal{S}$ denote the subclass of $\mathscr{A}$ consisting of univalent functions.

Suppose that $\mathscr{P}$ is the class of analytic functions $p$ normalized by

$$
p(z)=1+c_{1} z+c_{2} z^{2}+c_{3} z^{3}+\cdots
$$

and satisfying the condition

$$
\mathfrak{R}(p(z))>0(z \in \mathbb{D}) .
$$

Assume that $f$ and $g$ are two analytic functions in $\mathbb{D}$. Then, we say that the function $g$ is subordinate to the function $f$, and we write

$$
g(z) \prec f(z)(z \in \mathbb{D}),
$$

if there exists a Schwarz function $\omega(z)$ with $\omega(0)=0$ and $\mid \omega$ $(z) \mid<1$, such that (see [1])

$$
g(z)=f(\omega(z))(z \in \mathbb{D}) .
$$

In 2018, Cho et al. [2] introduced the following function class $S_{s}^{*}$ :

$$
S_{s}^{*}:=\left\{f \in \mathscr{A}: \frac{z f^{\prime}(z)}{f(z)} \prec(1+\sin z)(z \in \mathbb{D})\right\},
$$

which implies that the quantity $\left(z f^{\prime}(z)\right) /(f(z))$ lies in an eight-shaped region in the right-half plane.

In 1976, Noonan and Thomas [3] stated the $q^{\text {th }}$ Hankel determinant for $q \geq 1$ and $n \geq 1$ of functions $f$ as follows:

$$
H_{q}(n)=\left|\begin{array}{cccc}
a_{n} & a_{n+1} & \cdots & a_{n+q-1} \\
a_{n+1} & a_{n} & \cdots & a_{n+q-2} \\
\vdots & \vdots & & \vdots \\
a_{n+q-1} & a_{n+q-2} & \cdots & a_{n}
\end{array}\right|\left(a_{1}=1\right) .
$$


In particular, we have

$$
\begin{aligned}
& H_{2}(1)=\left|\begin{array}{ll}
a_{1} & a_{2} \\
a_{2} & a_{3}
\end{array}\right|=a_{3}-a_{2}^{2}\left(a_{1}=1, n=1, q=2\right), \\
& H_{2}(2)=\left|\begin{array}{ll}
a_{2} & a_{3} \\
a_{3} & a_{4}
\end{array}\right|=a_{2} a_{4}-a_{3}^{2}(n=2, q=2), \\
& H_{3}(1)=\left|\begin{array}{lll}
a_{1} & a_{2} & a_{3} \\
a_{2} & a_{3} & a_{4} \\
a_{3} & a_{4} & a_{5}
\end{array}\right|(n=1, q=3), \\
& H_{4}(1)=\left|\begin{array}{llll}
a_{1} & a_{2} & a_{3} & a_{4} \\
a_{2} & a_{3} & a_{4} & a_{4} \\
a_{3} & a_{4} & a_{5} & a_{6} \\
a_{4} & a_{5} & a_{6} & a_{7}
\end{array}\right| \quad(n=1, q=4) .
\end{aligned}
$$

Since $f \in \mathcal{S}, a_{1}=1$, thus

$$
\begin{aligned}
H_{4}(1)= & a_{7}\left\{a_{3}\left(a_{2} a_{4}-a_{3}^{2}\right)-a_{4}\left(a_{4}-a_{2} a_{3}\right)+a_{5}\left(a_{3}-a_{2}^{2}\right)\right\} \\
& -a_{6}\left\{a_{3}\left(a_{2} a_{5}-a_{3} a_{4}\right)-a_{4}\left(a_{5}-a_{2} a_{4}\right)+a_{6}\left(a_{3}-a_{2}^{2}\right)\right\} \\
& +a_{5}\left\{a_{3}\left(a_{3} a_{5}-a_{4}^{2}\right)-a_{5}\left(a_{5}-a_{2} a_{4}\right)+a_{6}\left(a_{4}-a_{2} a_{3}\right)\right\} \\
& +a_{5}\left\{a_{3}\left(a_{3} a_{5}-a_{4}^{2}\right)-a_{5}\left(a_{5}-a_{2} a_{4}\right)+a_{6}\left(a_{4}-a_{2} a_{3}\right)\right\} \\
& -a_{4}\left\{a_{4}\left(a_{3} a_{5}-a_{4}^{2}\right)-a_{5}\left(a_{2} a_{5}-a_{3} a_{4}\right)+a_{6}\left(a_{4}-a_{2} a_{3}\right)\right\} .
\end{aligned}
$$

We note that $H_{2}(1)$ is the well-known Fekete-Szegö functional (see [4-6]).

In recent years, many papers have been devoted to finding upper bounds for the second-order Hankel determinant $\mathrm{H}_{2}(2)$ and the third-order Hankel determinant $H_{3}(1)$, whose elements are various classes of analytic functions; it is worth mentioning that [7-20]. For instance, Murugusundaramoorthy and Bulboacă [21] defined a new subclass of analytic functions $M \boldsymbol{\Omega}_{c}^{a}(\lambda, \phi)$ and got upper bounds for the Fekete-Szegö functional and the Hankel determinant of order two for $f \in M \boldsymbol{Q}_{c}^{a}(\lambda, \phi)$. Islam et al. [22] examined the $q$-analog of starlike functions connected with a trigonometric sine function and discussed some interesting geometric properties, such as the well-known problems of Fekete-Szegö, the necessary and sufficient condition, the growth and distortion bound, closure theorem, and convolution results with partial sums for this class. Zaprawa et al. [23] obtained the bound of the third Hankel determinant for the univalent starlike functions. Very recently, Arif et al. [24] studied the problem of fourth Hankel determinant $H_{4}(1)$ for the first time for the class of bounded turning functions and successfully obtained the bound of $H_{4}(1)$. Recently, Khan et al. [25] discussed some classes of functions with bounded turning which are connected to the sine functions and obtained upper bounds for the third- and fourth-order Hankel determinants related to such classes. Inspired by the aforementioned works, in this paper, we mainly investigate upper bounds for the fourth- order Hankel determinant $H_{4}(1)$ for the function class $\mathcal{S}_{\mathcal{S}}^{*}$ associated with the sine function.

\section{Main Results}

By proving our desired results, we need the following lemmas.

Lemma 1 (see [26]). If $p(z) \in \mathscr{P}$, then exists some $x, z$ with $|x| \leq 1,|z| \leq 1$, such that

$$
\begin{aligned}
& 2 c_{2}=c_{1}^{2}+x\left(4-c_{1}^{2}\right), \\
& 4 c_{3}=c_{1}^{3}+2 c_{1} x\left(4-c_{1}^{2}\right)-\left(4-c_{1}^{2}\right) c_{1} x^{2}+2\left(4-c_{1}^{2}\right)\left(1-|x|^{2}\right) z .
\end{aligned}
$$

Lemma 2 (see [27]). Let $p(z) \in \mathscr{P}$, then

$$
\begin{aligned}
& \left|c_{1}^{4}+c_{2}^{2}+2 c_{1} c_{3}-3 c_{1}^{2} c_{2}-c_{4}\right| \leq 2, \\
& \left|c_{1}^{5}+3 c_{1} c_{2}^{2}+3 c_{1}^{2} c_{3}-4 c_{1}^{3} c_{2}-2 c_{1} c_{4}-2 c_{2} c_{3}+c_{5}\right| \leq 2, \\
& \mid c_{1}^{6}+6 c_{1}^{2} c_{2}^{2}+4 c_{1}^{3} c_{3}+2 c_{1} c_{5}+2 c_{2} c_{4}+c_{3}^{2}-c_{2}^{3} \\
& \quad-5 c_{1}^{4} c_{2}-3 c_{1}^{2} c_{4}-6 c_{1} c_{2} c_{3}-c_{6} \mid \leq 2 \\
& \left|c_{n}\right| \leq 2, n=1,2, \cdots .
\end{aligned}
$$

Lemma 3 (see [28]). Let $p(z) \in \mathscr{P}$, then we have

$$
\begin{aligned}
\left|c_{2}-\frac{c_{1}^{2}}{2}\right| & \leq 2-\frac{\left|c_{1}\right|^{2}}{2}, \\
\left|c_{n+k}-\mu c_{n} c_{k}\right| & <2, \quad 0 \leq \mu \leq 1, \\
\left|c_{n+2 k}-\mu c_{n} c_{k}^{2}\right| & \leq 2(1+2 \mu) .
\end{aligned}
$$

We now state and prove the main results of our present investigation.

Theorem 4. If the function $f(z) \in \mathcal{S}_{s}^{*}$ and of the form ((1)), then

$$
\begin{aligned}
& \left|a_{2}\right| \leq 1, \\
& \left|a_{3}\right| \leq \frac{1}{2}, \\
& \left|a_{4}\right| \leq 0.344, \\
& \left|a_{5}\right| \leq \frac{3}{8} \\
& \left|a_{6}\right| \leq \frac{67}{120}, \\
& \left|a_{7}\right| \leq \frac{5587}{10800} .
\end{aligned}
$$

Proof. Since $f(z) \in \mathcal{S}_{s}^{*}$, according to subordination relationship, thus there exists a Schwarz function $\omega(z)$ with $\omega(0)=$ 0 and $|\omega(z)|<1$, satisfying 


$$
\frac{z f^{\prime}(z)}{f(z)}=1+\sin (\omega(z))
$$

Here,

$$
\begin{aligned}
\frac{z f^{\prime}(z)}{f(z)=} & \frac{z+\sum_{n=2}^{\infty} n a_{n} z^{n}}{z+\sum_{n=2}^{\infty} a_{n} z^{n}}=\left(1+\sum_{n=2}^{\infty} n a_{n} z^{n-1}\right)\left[1-a_{2} z+\left(a_{2}^{2}-a_{3}\right) z^{2}\right. \\
& \left.-\left(a_{2}^{3}-2 a_{2} a_{3}+a_{4}\right) z^{3}+\left(a_{2}^{4}-3 a_{2}^{2} a_{3}+2 a_{2} a_{4}-a_{5}\right) z^{4}+\cdots\right] \\
= & 1+a_{2} z+\left(2 a_{3}-a_{2}^{2}\right) z^{2}+\left(a_{2}^{3}-3 a_{2} a_{3}+3 a_{4}\right) z^{3} \\
& +\left(4 a_{5}-a_{2}^{4}+4 a_{2}^{2} a_{3}-4 a_{2} a_{4}-2 a_{3}^{2}\right) z^{4} \\
& +\left(5 a_{6}-5 a_{2} a_{5}+a_{2}^{5}-5 a_{3} a_{4}-5 a_{2}^{3} a_{3}+5 a_{2}^{2} a_{4}+5 a_{2} a_{3}^{2}\right) z^{5} \\
& +\left(6 a_{7}-6 a_{2} a_{6}+6 a_{2}^{2} a_{5}-6 a_{3} a_{5}+12 a_{2} a_{3} a_{4}-a_{2}^{6}\right. \\
& \left.-6 a_{2}^{3} a_{4}-3 a_{4}^{2}+2 a_{3}^{3}-9 a_{2}^{2} a_{3}^{2}+6 a_{2}^{4} a_{3}\right) z^{6}+.
\end{aligned}
$$

Now, we define a function

$$
p(z)=\frac{1+\omega(z)}{1-\omega(z)}=1+c_{1} z+c_{2} z^{2}+\cdots
$$

It is easy to see that $p(z) \in \mathscr{P}$ and

$$
\omega(z)=\frac{p(z)-1}{1+p(z)}=\frac{c_{1} z+c_{2} z^{2}+c_{3} z^{3}+\cdots}{2+c_{1} z+c_{2} z^{2}+c_{3} z^{3}+\cdots} .
$$

On the other hand,

$$
\begin{aligned}
1+\sin (\omega(z))= & +\frac{1}{2} c_{1} z+\left(\frac{c_{2}}{2}-\frac{c_{1}^{2}}{4}\right) z^{2}+\left(\frac{5 c_{1}^{3}}{48}+\frac{c_{3}-c_{1} c_{2}}{2}\right) z^{3} \\
& +\left(\frac{c_{4}-c_{1} c_{3}}{2}+\frac{5 c_{1}^{2} c_{2}}{16}-\frac{c_{2}^{2}}{4}-\frac{c_{1}^{4}}{32}\right) z^{4} \\
& +\left(\frac{c_{5}-c_{1} c_{4}-c_{2} c_{3}}{2}+\frac{5 c_{1}^{2} c_{3}+c_{1} c_{2}^{2}}{16}-\frac{c_{1}^{3} c_{2}}{8}+\frac{c_{1}^{5}}{3840}\right) z^{5} \\
& +\left(\frac{c_{6}-c_{1} c_{5}-c_{2} c_{4}}{2}+\frac{5 c_{1} c_{2} c_{3}}{8}+\frac{5 c_{2}^{3}}{48}-\frac{c_{3}^{2}}{4}+\frac{5 c_{1}^{6}}{512}\right. \\
& \left.+\frac{c_{1}^{4} c_{2}}{768}-\frac{3 c_{1}^{2} c_{2}^{2}}{16}+\frac{5 c_{1}^{2} c_{4}}{16}-\frac{c_{1}^{3} c_{3}}{8}\right) z^{6}+\cdots
\end{aligned}
$$

Comparing the coefficients of $z, z^{2}, z^{3}, z^{4}, z^{5}, z^{6}$ between equations (15) and (18), we obtain

$$
\begin{aligned}
& a_{2}=\frac{c_{1}}{2}, \\
& a_{3}=\frac{c_{2}}{4}, \\
& a_{4}=\frac{c_{3}}{6}-\frac{c_{1} c_{2}}{24}-\frac{c_{1}^{3}}{144}, \\
& a_{5}=\frac{c_{4}}{8}-\frac{c_{1} c_{3}}{24}+\frac{5 c_{1}^{4}}{1152}-\frac{c_{1}^{2} c_{2}}{192}-\frac{c_{2}^{2}}{32}, \\
& a_{6}=\frac{-3 c_{1} c_{4}}{80}-\frac{7 c_{2} c_{3}}{120}-\frac{11 c_{1}^{5}}{4800}-\frac{43 c_{1} c_{2}^{2}}{960}+\frac{71 c_{1}^{3} c_{2}}{5760}+\frac{c_{5}}{10},
\end{aligned}
$$

$$
\begin{aligned}
a_{7}= & \frac{c_{1}^{2} c_{4}}{480}+\frac{c_{1} c_{2} c_{3}}{480}+\frac{833 c_{1}^{6}}{691200}-\frac{41 c_{1}^{2} c_{2}^{2}}{3840}-\frac{109 c_{1}^{4} c_{2}}{11520}-\frac{c_{1} c_{5}}{30} \\
& -\frac{5 c_{2} c_{4}}{96}+\frac{5 c_{2}^{3}}{1152}+\frac{c_{6}}{12}+\frac{c_{1}^{3} c_{3}}{144}
\end{aligned}
$$

Applying Lemma 2, we easily get

$$
\begin{aligned}
\left|a_{2}\right| & \leq 1, \\
\left|a_{3}\right| & \leq \frac{1}{2}, \\
\left|a_{4}\right|=\left|\frac{c_{3}}{6}-\frac{c_{1} c_{2}}{24}-\frac{c_{1}^{3}}{144}\right| & =\left|\frac{1}{6}\left[c_{3}-\frac{c_{1} c_{2}}{3}\right]+\frac{c_{1}}{72}\left[c_{2}-\frac{c_{1}^{2}}{2}\right]\right| .
\end{aligned}
$$

Let $c_{1}=c, c \in[0,2]$; by using Lemma 3 , we show

$$
\left|a_{4}\right|=\left|\frac{1}{6}\left[c_{3}-\frac{c_{1} c_{2}}{3}\right]+\frac{c_{1}}{72}\left[c_{2}-\frac{c_{1}^{2}}{2}\right]\right| \leq \frac{1}{3}+\frac{c\left(2-c^{2} / 2\right)}{72} ;
$$

also, let

$$
F(c)=\frac{1}{3}+\frac{c\left(2-c^{2} / 2\right)}{72}
$$

obviously, we find

$$
F^{\prime}(c)=\frac{1}{36}-\frac{c^{2}}{48}
$$

Setting $F^{\prime}(c)=0$, we have $c=2 \sqrt{3} / 3$, and so, $F(c)$ has a maximum value attained at $c=2 \sqrt{3} / 3$, also which is

$$
\begin{aligned}
\left|a_{4}\right| & \leq F\left(\frac{2 \sqrt{3}}{3}\right)=\frac{1}{3}+\frac{\sqrt{3}}{162} \approx 0.344, \\
\left|a_{5}\right| & =\left|\frac{c_{4}}{8}-\frac{c_{1} c_{3}}{24}+\frac{5 c_{1}^{4}}{1152}-\frac{c_{1}^{2} c_{2}}{192}-\frac{c_{2}^{2}}{32}\right| \\
& =\left|\frac{1}{8}\left[c_{4}-\frac{c_{1} c_{3}}{3}\right]-\frac{c_{1}^{2}}{576}\left[c_{2}-\frac{c_{1}^{2}}{2}\right]-\frac{c_{2}}{32}\left(c_{2}-\frac{c_{1}^{2}}{2}\right)-\frac{7 c_{1}^{2} c_{2}}{576}\right| .
\end{aligned}
$$

Let $c_{1}=c, c \in[0,2]$, according to Lemma 3, we obtain

$$
\left|a_{5}\right| \leq \frac{1}{4}+\frac{5 c^{2}\left(2-c^{2} / 2\right)}{576}+\frac{1}{16}\left(2-\frac{c^{2}}{2}\right)+\frac{7 c^{2}}{288} .
$$

Putting

$$
F(c)=\frac{1}{4}+\frac{5 c^{2}\left(2-c^{2} / 2\right)}{576}+\frac{1}{16}\left(2-\frac{c^{2}}{2}\right)+\frac{7 c^{2}}{288},
$$


we get

$$
F^{\prime}(c)=-\frac{7 c}{144}-\frac{5 c^{3}}{288} \leq 0
$$

Therefore, the function $F(c)$ has a maximum value attained at $c=0$, also which is

$$
\begin{aligned}
&\left|a_{5}\right| \leq F(0)=\frac{3}{8}, \\
&\left|a_{6}\right|=\left|\frac{-3 c_{1} c_{4}}{80}-\frac{7 c_{2} c_{3}}{120}-\frac{11 c_{1}^{5}}{4800}-\frac{43 c_{1} c_{2}^{2}}{960}+\frac{71 c_{1}^{3} c_{2}}{5760}+\frac{c_{5}}{10}\right| \\
&=\mid \frac{1}{24}\left[c_{5}-\frac{9 c_{1} c_{4}}{10}\right]+\frac{7}{120}\left[c_{5}-c_{2} c_{3}\right]+\frac{11 c_{1}^{3}}{2400}\left[c_{2}-\frac{c_{1}^{2}}{2}\right] \\
&-\frac{43 c_{1} c_{2}}{960}\left(c_{2}-\frac{c_{1}^{2}}{2}\right)-\frac{211 c_{1}^{3} c_{2}}{14400} \mid .
\end{aligned}
$$

Let $c_{1}=c, c \in[0,2]$, in view of Lemma 3, we have that

$$
\left|a_{6}\right| \leq \frac{7}{60}+\frac{1}{12}+\frac{11 c^{3}\left(2-c^{2} / 2\right)}{2400}+\frac{43}{240}\left(2-\frac{c^{2}}{2}\right)+\frac{211 c^{3}}{7200} \text {. }
$$

Taking

$F(c)=\frac{7}{60}+\frac{1}{12}+\frac{11 c^{3}\left(2-c^{2} / 2\right)}{2400}+\frac{43}{240}\left(2-\frac{c^{2}}{2}\right)+\frac{211 c^{3}}{7200}$,

we obtain

$$
F^{\prime}(c)=\frac{277 c^{2}}{2400}-\frac{55 c^{4}}{4800}-\frac{c}{240}
$$

Thus, $c=0$ is the root of the function $F^{\prime}(c)=0$ and $F^{\prime \prime}($ $0)<0$; we are easy to see that the function $F(c)$ has a maximum value attained at $c=0$, also which is

$$
\begin{aligned}
&\left|a_{6}\right| \leq F(0)=\frac{67}{120}, \\
&\left|a_{7}\right|=\mid \frac{c_{1}^{2} c_{4}}{480}+\frac{c_{1} c_{2} c_{3}}{480}+\frac{833 c_{1}^{6}}{691200}-\frac{41 c_{1}^{2} c_{2}^{2}}{3840}-\frac{109 c_{1}^{4} c_{2}}{11520} \\
&-\frac{c_{1} c_{5}}{30}-\frac{5 c_{2} c_{4}}{96}+\frac{5 c_{2}^{3}}{1152}+\frac{c_{6}}{12}+\frac{c_{1}^{3} c_{3}}{144} \mid \\
&= \mid \frac{-37 c_{1}^{6}}{691200}-\frac{25 c_{1}^{2} c_{2}^{2}}{5760}-\frac{c_{1} c_{5}}{30}+\frac{c_{1}^{2}\left[c_{4}-c_{2}^{2}\right]}{480}+\frac{c_{1} c_{2}\left[c_{3}-c_{1} c_{2}\right]}{480} \\
&+\frac{c_{1}^{3}\left[c_{3}-c_{1} c_{2}\right]}{144}-\frac{29 c_{1}^{4}\left[c_{2}-c_{1}^{2} / 2\right]}{11520}+\frac{5 c_{2}^{2}\left[c_{2}-c_{1}^{2} / 2\right]}{1152} \\
&+\frac{\left[c_{6}-5 / 8 c_{2} c_{4}\right]}{12} \mid .
\end{aligned}
$$

Let $c_{1}=c, c \in[0,2]$, by virtue of Lemma 3, we have that

$$
\begin{aligned}
\left|a_{7}\right| \leq & \frac{1}{6}+\frac{c^{2}}{240}+\frac{9 c}{120}+\frac{29 c^{4}\left(2-c^{2} / 2\right)}{11520}+\frac{37 c^{6}}{691200}+\frac{c^{3}}{72} \\
& +\frac{25 c^{2}}{1440}+\frac{5\left(2-c^{2} / 2\right)}{288} .
\end{aligned}
$$

Letting

$$
\begin{aligned}
F(c)= & \frac{1}{6}+\frac{c^{2}}{240}+\frac{9 c}{120}+\frac{29 c^{4}\left(2-c^{2} / 2\right)}{11520}+\frac{37 c^{6}}{691200}+\frac{c^{3}}{72} \\
& +\frac{25 c^{2}}{1440}+\frac{5\left(2-c^{2} / 2\right)}{288},
\end{aligned}
$$

so we get

$$
F^{\prime}(c) \geq 0
$$

Thus, the function $F(c)$ has a maximum value attained at $c=2$, also which is

$$
\left|a_{7}\right| \leq F(2)=\frac{5587}{10800}
$$

Hence, the proof is complete.

Theorem 5. If the function $f(z) \in \mathcal{S}_{s}^{*}$ and of the form ((1)), then we have

$$
\left|a_{3}-a_{2}^{2}\right| \leq \frac{1}{2}
$$

Proof. Applying equation (21), we have

$$
\left|a_{3}-a_{2}^{2}\right|=\left|\frac{c_{2}}{4}-\frac{c_{1}^{2}}{4}\right|
$$

Then, by applying Lemma 1, we get

$$
\left|a_{3}-a_{2}^{2}\right|=\left|\frac{x\left(4-c_{1}^{2}\right)}{8}-\frac{c_{1}^{2}}{8}\right|
$$

Suppose that $|x|=t, t \in[0,1], c_{1}=c, c \in[0,2]$. Then, using the triangle inequality, we obtain

$$
\left|a_{3}-a_{2}^{2}\right| \leq \frac{t\left(4-c^{2}\right)}{8}+\frac{c^{2}}{8}
$$

Suppose

$$
F(c, t)=\frac{t\left(4-c^{2}\right)}{8}+\frac{c^{2}}{8}
$$

then for any $t \in(0,1)$ and $c \in(0,2)$, we get 


$$
\frac{\partial F}{\partial t}=\frac{4-c^{2}}{8}>0
$$

which means that $F(c, t)$ is an increasing function on the closed interval $[0,1]$ about $t$. Therefore, the function $F(c, t)$ can get the maximum value at $t=1$, that is,

$$
\max F(c, t)=F(c, 1)=\frac{\left(4-c^{2}\right)}{8}+\frac{c^{2}}{8}=\frac{1}{2} .
$$

So, obviously,

$$
\left|a_{3}-a_{2}^{2}\right| \leq \frac{1}{2}
$$

Hence, the proof is complete.

Theorem 6. If the function $f(z) \in \mathcal{S}_{s}^{*}$ and of the form ((1)), then we have

$$
\left|a_{2} a_{3}-a_{4}\right| \leq \frac{1}{3}
$$

Proof. From (21), we have

$$
\left|a_{2} a_{3}-a_{4}\right|=\left|\frac{c_{1} c_{2}}{8}+\frac{c_{1}^{3}}{144}-\frac{c_{3}}{6}+\frac{c_{1} c_{2}}{24}\right|=\left|\frac{c_{1} c_{2}}{6}-\frac{c_{3}}{6}+\frac{c_{1}^{3}}{144}\right| .
$$

Now, in view of Lemma 1, we get

$$
\left|a_{2} a_{3}-a_{4}\right|=\left|\frac{7 c_{1}^{3}}{144}+\frac{\left(4-c_{1}^{2}\right) c_{1} x^{2}}{24}-\frac{\left(4-c_{1}^{2}\right)\left(1-|x|^{2}\right) z}{12}\right|
$$

Let $|x|=t, t \in[0,1], c_{1}=c, c \in[0,2]$. Then, using the triangle inequality, we deduce that

$$
\left|a_{2} a_{3}-a_{4}\right| \leq \frac{7 c^{3}}{144}+\frac{\left(4-c^{2}\right) c t^{2}}{24}+\frac{\left(4-c^{2}\right)\left(1-t^{2}\right)}{12} .
$$

Assume that

$$
F(c, t)=\frac{7 c^{3}}{144}+\frac{\left(4-c^{2}\right) c t^{2}}{24}+\frac{\left(4-c^{2}\right)\left(1-t^{2}\right)}{12} .
$$

Therefore, for any $t \in(0,1)$ and $c \in(0,2)$, we have

$$
\frac{\partial F}{\partial t}=\frac{\left(4-c^{2}\right) t(c-2)}{12}<0
$$

that is, $F(c, t)$ is an decreasing function on the closed interval $[0,1]$ about $t$. This implies that the maximum value of $F(c, t)$ occurs at $t=0$, which is

$$
\max F(c, t)=F(c, 0)=\frac{7 c^{3}}{144}+\frac{\left(4-c^{2}\right)}{12} .
$$

Define

$$
G(c)=\frac{\left(4-c^{2}\right)}{12}+\frac{7 c^{3}}{144}
$$

we clearly see that the function $G(c)$ has a maximum value attained at $c=0$, also which is

$$
\left|a_{2} a_{3}-a_{4}\right| \leq G(0)=\frac{1}{3}
$$

Hence, the proof is complete.

Theorem 7. If the function $f(z) \in \mathcal{S}_{s}^{*}$ and of the form ((1)), then we have

$$
\left|a_{2} a_{4}-a_{3}^{2}\right| \leq \frac{1}{4}
$$

Proof. Let $f(z) \in \mathcal{S}_{s}^{*}$, then by equation (21), we get

$$
\left|a_{2} a_{4}-a_{3}^{2}\right|=\left|\frac{c_{1} c_{3}}{12}-\frac{c_{1}^{2} c_{2}}{48}-\frac{c_{1}^{4}}{288}-\frac{c_{2}^{2}}{16}\right|
$$

Now, in terms of Lemma 1, we obtain

$$
\begin{aligned}
\left|a_{2} a_{4}-a_{3}^{2}\right|= & \left|\frac{c_{1} c_{3}}{12}-\frac{c_{1}^{2} c_{2}}{48}-\frac{c_{1}^{4}}{288}-\frac{c_{2}^{2}}{16}\right| \\
= & \mid-\frac{5 c_{1}^{4}}{576}-\frac{x^{2} c_{1}^{2}\left(4-c_{1}^{2}\right)}{48}-\frac{x^{2}\left(4-c_{1}^{2}\right)^{2}}{64} \\
& +\frac{c_{1}\left(4-c_{1}^{2}\right)\left(1-|x|^{2}\right) z}{24} \mid .
\end{aligned}
$$

Let $|x|=t, t \in[0,1], c_{1}=c, c \in[0,2]$. Then, using the triangle inequality, we get

$\left|a_{2} a_{4}-a_{3}^{2}\right| \leq \frac{t^{2} c^{2}\left(4-c^{2}\right)}{48}+\frac{\left(1-t^{2}\right) c\left(4-c^{2}\right)}{24}+\frac{t^{2}\left(4-c^{2}\right)^{2}}{64}+\frac{5 c^{4}}{576}$

Setting

$F(c, t)=\frac{t^{2} c^{2}\left(4-c^{2}\right)}{48}+\frac{\left(1-t^{2}\right) c\left(4-c^{2}\right)}{24}+\frac{t^{2}\left(4-c^{2}\right)^{2}}{64}+\frac{5 c^{4}}{576}$,

then, for any $t \in(0,1)$ and $c \in(0,2)$, we have

$$
\frac{\partial F}{\partial t}=\frac{t\left(c^{2}-8 c+12\right)\left(4-c^{2}\right)}{96}>0,
$$

which implies that $F(c, t)$ increases on the closed interval $[0,1]$ about $t$. That is, that $F(c, t)$ has a maximum value at $t=1$, which is 
$\max F(c, t)=F(c, 1)=\frac{c^{2}\left(4-c^{2}\right)}{48}+\frac{\left(4-c^{2}\right)^{2}}{64}+\frac{5 c^{4}}{576}$.

Putting

$$
G(c)=\frac{c^{2}\left(4-c^{2}\right)}{48}+\frac{\left(4-c^{2}\right)^{2}}{64}+\frac{5 c^{4}}{576},
$$

then we have

$$
G^{\prime}(c)=\frac{c\left(4-c^{2}\right)}{24}-\frac{c^{3}}{24}-\frac{c\left(4-c^{2}\right)}{16}+\frac{5 c^{3}}{144}
$$

If $G^{\prime}(c)=0$, then the root is $c=0$. Also, since $G^{\prime \prime}(0)=$ $-1 / 12<0$, so the function $G(c)$ can take the maximum value at $c=0$, which is

$$
\left|a_{2} a_{4}-a_{3}^{2}\right| \leq G(0)=\frac{1}{4}
$$

Hence, the proof is complete.

Theorem 8. If the function $f(z) \in \mathcal{S}_{s}^{*}$ and of the form ((1)), then we have

$$
\left|a_{2} a_{5}-a_{3} a_{4}\right| \leq \frac{11}{36}
$$

Proof. Let $f(z) \in \mathcal{S}_{s}^{*}$, then by using (21), we have

$$
\begin{aligned}
\left|a_{2} a_{5}-a_{3} a_{4}\right|= & \left|\frac{5 c_{1}^{5}}{2304}+\frac{c_{1} c_{4}}{16}-\frac{c_{1} c_{2}^{2}}{192}-\frac{c_{1}^{2} c_{3}}{48}-\frac{c_{1}^{3} c_{2}}{1152}-\frac{c_{2} c_{3}}{24}\right| \\
= & \mid-\frac{c_{1}^{3}\left[c_{2}-c_{1}^{2} / 2\right]}{1152}-\frac{c_{3}\left[c_{2}-c_{1}^{2} / 2\right]}{24}+\frac{c_{1}\left[c_{4}-c_{1} c_{3}\right]}{24} \\
& +\frac{c_{1}^{5}}{576}+\frac{c_{1}\left[c_{4}-1 / 4 c_{2}^{2}\right]}{48} \mid .
\end{aligned}
$$

Let $c_{1}=c, c \in[0,2]$, according to Lemma 3 , we obtain

$$
\left|a_{5}\right| \leq \frac{1}{4}+\frac{5 c^{2}\left(2-c^{2} / 2\right)}{576}+\frac{1}{16}\left(2-\frac{c^{2}}{2}\right)+\frac{7 c^{2}}{288}
$$

Taking

$$
F(c)=\frac{c^{3}\left[2-c^{2} / 2\right]}{1152}+\frac{\left[2-c^{2} / 2\right]}{12}+\frac{c}{8}+\frac{c^{5}}{576} .
$$

Then, $\forall c \in(0,2)$, we have

$$
F^{\prime}(c)=\frac{c^{2}}{192}+\frac{c^{4}}{128}-\frac{c}{12}+\frac{1}{8}>0
$$

which implies that $F(c)$ increases on the closed interval $[0,2]$ about $c$. Namely, the maximum value of $F(c)$ attains at $c=2$, also which is

$$
\left|a_{2} a_{5}-a_{3} a_{4}\right| \leq F(2)=\frac{11}{36}
$$

The proof of Theorem 8 is completed.

Theorem 9. If the function $f(z) \in \mathcal{S}_{s}^{*}$ and of the form ((1)), then we have

$$
\left|a_{5}-a_{2} a_{4}\right| \leq \frac{13}{32}
$$

Proof. Assume that $f(z) \in \mathcal{S}_{s}^{*}$, then from (21), we obtain

$$
\begin{aligned}
\left|a_{5}-a_{2} a_{4}\right|= & \left|\frac{c_{1}^{4}}{128}-\frac{c_{1} c_{3}}{8}+\frac{c_{1}^{2} c_{2}}{64}-\frac{c_{2}^{2}}{32}+\frac{c_{4}}{8}\right| \\
= & \mid \frac{\left[c_{1}^{4}+c_{2}^{2}+2 c_{1} c_{3}-3 c_{1}^{2} c_{2}-c_{4}\right]}{32}-\frac{5 c_{1}^{2}\left[c_{2}-c_{1}^{2} / 2\right]}{64} \\
& -\frac{3\left[c_{4}-2 / 3 c_{1} c_{3}\right]}{32} \mid .
\end{aligned}
$$

Next, by virtue of Lemma 3, we obtain

$$
\left|a_{5}-a_{2} a_{4}\right| \leq \frac{1}{4}+\frac{5 c^{2}\left[2-c^{2} / 2\right]}{64}
$$

Setting

$$
F(c)=\frac{1}{4}+\frac{5 c^{2}\left[2-c^{2} / 2\right]}{64}
$$

Then, we have

$$
F^{\prime}(c)=\frac{5 c}{16}-\frac{5 c^{3}}{32}
$$

Let $F^{\prime}(c)=0$, we get $c=0$ or $c=\sqrt{2}$ and $F^{\prime}(\sqrt{2})<0$, which implies that the maximum value of $F(c)$ attains at $c$ $=\sqrt{2}$, also which is

$$
\left|a_{5}-a_{2} a_{4}\right| \leq F(\sqrt{2})=\frac{13}{32} .
$$

Hence, the proof is complete.

Theorem 10. If the function $f(z) \in \mathcal{S}_{s}^{*}$ and of the form ((1)), then we have

$$
\left|a_{5} a_{3}-a_{4}^{2}\right| \leq \frac{97}{324}
$$


Proof. Assume that $f(z) \in \mathcal{S}_{s}^{*}$, then from (21), we obtain

$$
\begin{aligned}
\left|a_{5} a_{3}-a_{4}^{2}\right|= & \mid \frac{7 c_{1}^{4} c_{2}}{13824}+\frac{c_{2} c_{4}}{32}+\frac{c_{1} c_{2} c_{3}}{288}-\frac{c_{2}^{3}}{128}+\frac{c_{1}^{3} c_{3}}{432} \\
& -\frac{7 c_{1}^{2} c_{2}^{2}}{2304}-\frac{c_{3}^{2}}{36}-\frac{c_{1}^{6}}{20736} \mid \\
= & \mid \frac{c_{2}\left[c_{4}-c_{1} c_{3} / 9\right]}{32}-\frac{c_{3}\left[c_{3}-c_{1} c_{2} / 4\right]}{36}-\frac{c_{2}^{2}\left[c_{2}-c_{1}^{2} / 2\right]}{128} \\
& -\frac{c_{1}^{2} c_{2}\left[c_{2}-c_{1}^{2} / 2\right]}{144}+\frac{c_{1}^{3}\left[c_{3}-31 / 32 c_{1} c_{2}\right]}{432} \\
& -\frac{5 c_{1}^{4} c_{2}}{6912}-\frac{c_{1}^{6}}{20736} \mid .
\end{aligned}
$$

Next, in terms of Lemma 3, we obtain

$$
\begin{aligned}
\left|a_{5} a_{3}-a_{4}^{2}\right| \leq & \frac{1}{8}+\frac{1}{9}+\frac{\left[2-c^{2} / 2\right]}{32}+\frac{c^{2}\left[2-c^{2} / 2\right]}{72}+\frac{c^{3}}{216} \\
& +\frac{5 c^{4}}{3456}+\frac{c^{6}}{20736} .
\end{aligned}
$$

Putting

$$
\begin{aligned}
F(c)= & \frac{1}{8}+\frac{1}{9}+\frac{\left[2-c^{2} / 2\right]}{32}+\frac{c^{2}\left[2-c^{2} / 2\right]}{72}+\frac{c^{3}}{216} \\
& +\frac{5 c^{4}}{3456}+\frac{c^{6}}{20736} .
\end{aligned}
$$

Then, for any $c \in(0,2)$, we have $F^{\prime}(c)>0$, which means that the maximum value of $F(c)$ arrives at $t=2$, also which is

$$
\left|a_{5} a_{3}-a_{4}^{2}\right| \leq F(2)=\frac{97}{324} .
$$

Hence, the proof is complete.

Theorem 11. If the function $f(z) \in \mathcal{S}_{s}^{*}$ and of the form ((1)), then we have

$$
\left|H_{4}(1)\right| \leq 0.81945 .
$$

Proof. Because of

$$
\begin{aligned}
H_{4}(1)= & a_{7}\left\{a_{3}\left(a_{2} a_{4}-a_{3}^{2}\right)-a_{4}\left(a_{4}-a_{2} a_{3}\right)+a_{5}\left(a_{3}-a_{2}^{2}\right)\right\} \\
& -a_{6}\left\{a_{3}\left(a_{2} a_{5}-a_{3} a_{4}\right)-a_{4}\left(a_{5}-a_{2} a_{4}\right)+a_{6}\left(a_{3}-a_{2}^{2}\right)\right\} \\
& -a_{6}\left\{a_{3}\left(a_{2} a_{5}-a_{3} a_{4}\right)-a_{4}\left(a_{5}-a_{2} a_{4}\right)+a_{6}\left(a_{3}-a_{2}^{2}\right)\right\} \\
& +a_{5}\left\{a_{3}\left(a_{3} a_{5}-a_{4}^{2}\right)-a_{5}\left(a_{5}-a_{2} a_{4}\right)+a_{6}\left(a_{4}-a_{2} a_{3}\right)\right\} \\
& -a_{4}\left\{a_{4}\left(a_{3} a_{5}-a_{4}^{2}\right)-a_{5}\left(a_{2} a_{5}-a_{3} a_{4}\right)+a_{6}\left(a_{4}-a_{2} a_{3}\right)\right\},
\end{aligned}
$$

then, by applying the triangle inequality, we get

$$
\begin{aligned}
\left|H_{4}(1)\right|= & \left|a_{7}\right|\left|a_{3}\right||| a_{2} a_{4}-a_{3}^{2}|+| a_{7}|| a_{4}|| a_{4}-a_{2} a_{3} \mid \\
& +\left|a_{7}\right|\left|a_{5}\right|\left|a_{3}-a_{2}^{2}\right|+\left|a_{6}\right|\left|a_{3}\right|\left|a_{2} a_{5}-a_{3} a_{4}\right| \\
& +\left|a_{6}\right|\left|a_{4}\right|\left|a_{5}-a_{2} a_{4}\right|+\left|a_{6}\right|^{2}\left|a_{3}-a_{2}^{2}\right| \\
& +\left|a_{5}\right|\left|a_{3}\right|\left|a_{3} a_{5}-a_{4}^{2}\right|+\left|a_{5}\right|^{2}\left|a_{5}-a_{2} a_{4}\right| \\
& +\left|a_{5}\right|\left|a_{6}\right|\left|a_{4}-a_{2} a_{3}\right|+\left|a_{4}\right|^{2}\left|a_{3} a_{5}-a_{4}^{2}\right| \\
& +\left|a_{4}\right|\left|a_{5}\right|\left|a_{2} a_{5}-a_{3} a_{4}\right|+\left|a_{4}\right|\left|a_{6}\right|\left|a_{4}-a_{2} a_{3}\right| .
\end{aligned}
$$

Next, substituting (13) and (39)-(78) into (85), we easily obtain the desired assertion (83).

\section{Conclusion}

In the present paper, we mainly get upper bounds of the fourth-order Hankel determinant $H_{4}(1)$ of starlike functions connected with the sine function. However, the results obtained in this paper are not sharp. In the future, we will consider the sharpness of the results. Also, we can discuss the related research of the fifth-order Hankel determinant and fifth-order Toeplitz determinant for this function class.

\section{Data Availability}

No data were used to support this study.

\section{Conflicts of Interest}

The authors declare that they have no conflicts of interest.

\section{Acknowledgments}

The present investigation was partly supported by the National Natural Science Foundation of the People's Republic of China under Grant 11561001, the Program for Young Talents of Science and Technology in Universities of Inner Mongolia Autonomous Region under Grant NJYT-18-A14, the Natural Science Foundation of Inner Mongolia of the People's Republic of China under Grant 2018MS01026, and the Higher School Foundation of Inner Mongolia of the People's Republic of China under Grant NJZY20200.

\section{References}

[1] S. S. Miller and P. T. Mocanu, Differential Subordinations: Theory and Applications, Series on Monographs and Textbooks in Pure and Applied Mathematics, no. 225, 2000CRC Press, New York and Basel, 2000.

[2] N. E. Cho, V. Kumar, S. S. Kumar, and V. Ravichandran, "Radius problems for starlike functions associated with the sine function," Bulletin of the Iranian Mathematical Society, vol. 45, no. 1, pp. 213-232, 2019.

[3] J. W. Noonan and D. K. Thomas, "On the second Hankel determinant of areally mean p-valent functions," Transactions of the American Mathematical Society, vol. 223, no. 2, pp. 337$346,1976$. 
[4] M. Fekete and G. Szegö, "Eine Bemerkung Über Ungerade schlichte Funktionen," Journal of the london mathematical society, vol. s1-8, no. 2, pp. 85-89, 1933.

[5] W. Koepf, "On the Fekete-Szego problem for close-to-convex functions," Proceedings of the American Mathematical Society, vol. 101, pp. 89-95, 1987.

[6] W. Koepf, "On the Fekete-Szego problem for close-to-convex functions II," Archiv der Mathematik, vol. 49, no. 5, pp. 420433, 1987.

[7] D. K. Thomas and S. Abdul Halim, "Retracted article: Toeplitz matrices whose elements are the coefficients of starlike and close-to-convex functions," Bulletin of the Malaysian Mathematical Sciences Society, vol. 40, no. 4, article 385, pp. 17811790, 2017.

[8] K. O. Babalola, "On $\mathrm{H}_{3}$ (1) Hankel determinant for some classes of univalent functions," in Inequality Theory and Applications, Y. J. Cho, J. K. Kim, and S. S. Dragomir, Eds., vol. 6, pp. 1-7, University of Ilorin, 2010.

[9] D. Bansal, "Upper bound of second Hankel determinant for a new class of analytic functions," Applied Mathematics Letters, vol. 26, no. 1, pp. 103-107, 2013.

[10] D. Bansal, S. Maharana, and J. K. Prajapat, "Third order Hankel determinant for certain univalent functions," Journal of the Korean Mathematical Society, vol. 52, no. 6, pp. 1139-1148, 2015.

[11] A. Janteng, S. Halim, and M. Darus, "Coefficient inequality for a function whose derivative has a positive real part," Journal of Inequalities in Pure and Applied Mathematics, vol. 7, no. 2, 2006.

[12] S. Janteng, A. Halim, and M. Darus, "Hankel determinant for starlike and convex functions," International Journal of Mathematical Analysis, vol. 13, no. 1, pp. 619-625, 2007.

[13] S. K. Lee, V. Ravichandran, and S. Supramaniam, "Bounds for the second Hankel determinant of certain univalent functions," Journal of inequalities and Applications, vol. 2013, no. 1, Article ID 729, 2013.

[14] M. Raza and S. N. Malik, "Upper bound of the third Hankel determinant for a class of analytic functions related with lemniscate of Bernoulli," Journal of Inequalities and Applications, vol. 2013, no. 1, Article ID 2378, 8 pages, 2013.

[15] P. Zaprawa, "Third Hankel determinants for subclasses of univalent functions," Mediterranean Journal of Mathematics, vol. 14, no. 1, 2017.

[16] H. Y. Zhang, H. Tang, and L. N. Ma, "Upper bound of third Hankel determinant for a class of analytic functions," Pure and Applied Mathematics, vol. 33, no. 2, pp. 211-220, 2017.

[17] S. Mahmood, H. M. Srivastava, N. Khan, Q. Z. Ahmad, B. Khan, and I. Ali, "Upper bound of the third Hankel determinant for a subclass of q-starlike functions," Symmetry, vol. 11, no. 3, p. 347, 2019.

[18] L. Shi, I. Ali, M. Arif, N. E. Cho, S. Hussain, and H. Khan, “A study of third Hankel determinant problem for certain subfamilies of analytic functions involving cardioid domain," Mathematics, vol. 7, no. 5, p. 418, 2019.

[19] L. Shi, H. M. Srivastava, M. Arif, S. Hussain, and H. Khan, "An investigation of the third Hankel determinant problem for certain subfamilies of univalent functions involving the exponential function," Symmetry, vol. 11, no. 5, p. 598, 2019.

[20] S. K. Lee, K. Khatter, and V. Ravichandran, "Radius of starlikeness for classes of analytic functions," Bulletin of the Malaysian Mathematical Sciences Society, vol. 43, no. 6, article 1028, pp. 4469-4493, 2020.
[21] G. Murugusundaramoorthy and T. Bulboacă, "Hankel determinants for new subclasses of analytic functions related to a shell shaped region," Mathematics, vol. 8, no. 6, p. 1041, 2020.

[22] S. Islam, M. G. Khan, B. Ahmad, M. Arif, and R. Chinram, "Qextension of starlike functions subordinated with a trigonometric sine function," Mathematics, vol. 8, no. 10, p. 1676, 2020.

[23] P. Zaprawa, M. Obradović, and N. Tuneski, "Third Hankel determinant for univalent starlike functions," Revista de la Real Academia de Ciencias Exactas, Físicas y Naturales. Serie A. Matemáticas, vol. 115, no. 2, pp. 1-6, 2021.

[24] M. Arif, L. Rani, M. Raza, and P. Zaprawa, "Fourth Hankel determinant for the family of functions with bounded turning," Bulletin of the Korean Mathematical Society, vol. 55, no. 6, pp. 1703-1711, 2018.

[25] M. G. Khan, B. Ahmad, J. Sokol et al., "Coefficient problems in a class of functions with bounded turning associated with sine function," European Journal of Pure and Applied Mathematics, vol. 14, no. 1, pp. 53-64, 2021.

[26] R. J. Libera and E. J. Zlotkiewicz, "Coefficient bounds for the inverse of a function with derivative in $\mathrm{P}$," Proceedings of the American Mathematical Society, vol. 87, no. 2, pp. 251-257, 1983.

[27] V. Ravichandran and S. Verma, "Bound for the fifth coefficient of certain starlike functions," Comptes Rendus Mathematique, vol. 353, no. 6, pp. 505-510, 2015.

[28] C. Pommerenke, "Univalent functions," in Mathematik, Lehrbucher, vandenhoeck and Ruprecht, Gottingen, 1975. 